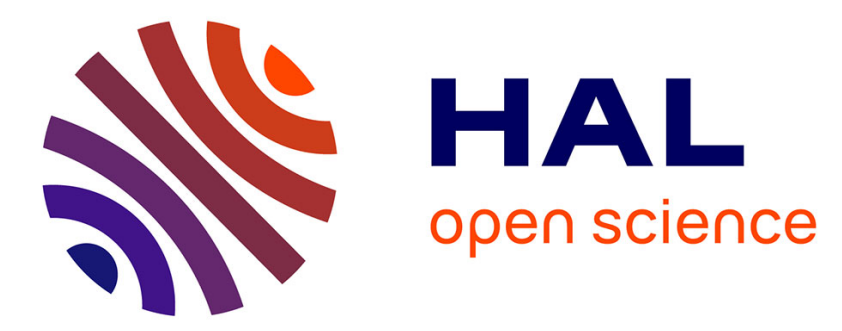

\title{
FID navigator-based MR thermometry method to monitor small temperature changes in the brain of ventilated animals
}

Nicolas Boulant, Michel Bottlaender, Lynn Uhrig, Eric Giacomini, Michel Luong, Alexis Amadon, Aurélien Massire, Benoit Larrat, Alexandre Vignaud

\section{To cite this version:}

Nicolas Boulant, Michel Bottlaender, Lynn Uhrig, Eric Giacomini, Michel Luong, et al.. FID navigator-based MR thermometry method to monitor small temperature changes in the brain of ventilated animals. NMR in Biomedicine, 2014, 28, pp.101-107. 10.1002/nbm.3232 . cea-02043287

\section{HAL Id: cea-02043287 https://hal-cea.archives-ouvertes.fr/cea-02043287}

Submitted on 20 Feb 2019

HAL is a multi-disciplinary open access archive for the deposit and dissemination of scientific research documents, whether they are published or not. The documents may come from teaching and research institutions in France or abroad, or from public or private research centers.
L'archive ouverte pluridisciplinaire HAL, est destinée au dépôt et à la diffusion de documents scientifiques de niveau recherche, publiés ou non, émanant des établissements d'enseignement et de recherche français ou étrangers, des laboratoires publics ou privés. 


\title{
FID navigator-based MR thermometry method to monitor small temperature changes in the brain of ventilated animals
}

\author{
Nicolas Boulant ${ }^{a *}$, Michel Bottlaender $^{b}$, Lynn Uhrig ${ }^{b}$, Eric Giacomini ${ }^{a}$, \\ Michel Luong ${ }^{c}$, Alexis Amadon ${ }^{a}$, Aurélien Massire ${ }^{a}$, Benoît Larrat ${ }^{a}$ \\ and Alexandre Vignaud ${ }^{\mathrm{a}}$
}

\begin{abstract}
An MR thermometry method is proposed for measuring in vivo small temperature changes engendered by external RF heat sources. The method relies on reproducible and stable respiration and therefore currently applies to ventilated animals whose breathing is carefully controlled. It first consists in characterizing the stability of the main magnetic field as well as the variations induced by breathing during a first monitoring stage. Second, RF heating is applied while the phase and thus temperature evolutions are continuously measured, the corrections due to breathing and field drift being made thanks to the data accumulated during the first period. The RF heat source is finally stopped and the temperature rise likewise is continuously monitored during a third and last stage to observe the animal cooling down and to validate the assumptions made for correcting for the main field variation and the physiological noise. Experiments were performed with a clinical $7 \mathrm{~T}$ scanner on an anesthetized baboon and with a dedicated RF heating setup. Analysis of the data reveals a precision around $0.1^{\circ} \mathrm{C}$, which allows us to reliably measure sub-degree temperature rises in the muscle and in the brain of the animal. Copyright ๑ 2014 John Wiley \& Sons, Ltd.
\end{abstract}

Keywords: MR thermometry; proton resonance frequency shift; navigator FID

\section{INTRODUCTION}

Non-invasive monitoring of temperature rises using MR thermometry (MRT) benefits from ultra-high field (UHF) due to an increased signal-to-noise-ratio as well as an increased sensitivity of the proton resonance frequency shift (PRFS) method. First observed in 1966 by Hindman (1), the method consists in exploiting the small and linear frequency shift locally experienced by the nuclear spins due to corresponding changes of the fraction of hydrogen bonds and of the magnetic susceptibility with respect to temperature (2). The former phenomenon dominating the latter by almost an order of magnitude, the total shift of the resonance frequency can be considered as tissue-type independent to first order (except for adipose tissues) and equal to $-0.01 \mathrm{ppm} /{ }^{\circ} \mathrm{C}$ (2). Combined with the linearity of the phenomenon and the sensitivity of the technique, the PRFS method has been a very valuable tool for monitoring temperature variations in vivo. Phase mapping techniques however suffer from field drifts, motion and physiological noise sources such as breathing and heartbeat, which are amplified at UHF. Partial correction of these effects can be done via a variety of techniques (2). Moreover when the heat source is highly localized, as with high intensity focused ultrasound (HIFU) techniques (3-7), referencefree methods $(8,9)$ are particularly attractive, since all acquired phase images are self-contained and do not require subtraction from a different reference image, thereby making the data more immune to respiration and motion. In this case the temperature rise is monitored in order to control accurately the thermal dose deposited in the targeted tissue during a possible MR-guided focused ultrasound ablation.
With the evolution of magnetic field strengths and RF coil technologies, the relation between temperature and the specific absorption rate (SAR) has been of recent interest in the MRI community (10-13). A better understanding of their relation however requires more experiments and robust tools to measure noninvasively small temperature rises induced by RF, non-localized, heat sources. Likewise, because typical RF powers used by imaging coils deposit much less energy than HIFU, longer heating periods must be applied to observe a temperature rise of the order of $1{ }^{\circ} \mathrm{C}$, thereby adding constraints on the system's stability. In addition, regardless of the amount of energy deposit, such longer periods may be necessary to observe with confidence the time-scales involved in some bio-heat models $(12,14)$. Reliable sequences and post-processing methods able to extract

\footnotetext{
* Correspondence to: Nicolas Boulant, CEA/DSV/I2BM/NeuroSpin/UNIRS, CEA Saclay, 91191 Gif sur Yvette, France.

E-mail: nicolas.boulant@cea.fr

a N. Boulant, E. Giacomini, A. Amadon, A. Massire, B. Larrat, A. Vignaud CEA/DSV/I2BM/NeuroSpin/UNIRS, CEA Saclay, 91191 Gif sur Yvette, France

b M. Bottlaender, L. Uhrig CEA/DSV/I2BM/NeuroSpin/UNIACT, CEA Saclay, 91191 Gif sur Yvette, France

c M. Luong

CEA/DSM/Irfu/SACM, CEA Saclay, 91191 Gif sur Yvette, France
}

Abbreviations used: HIFU, high intensity focused ultrasound; MRT, magnetic
resonance thermometry; UHF, ultra-high field; FID, free induction decay; PRFS,
proton resonance frequency shift; SAR, specific absorption rate; GRE, gradient recalled echo. 
the relevant information from the noise are then needed. In this work, we report a new method based on navigator free induction decays (FIDs) for monitoring in vivo small temperature rises such as those typically encountered in MRI exams. Although the method applies to any type of heat source, to provide further control and stability the source in this study is an external RF field producing a smeared energy deposit (thus ruling out the use of reference-free methods). In addition, due to the methodology employed, the technique so far applies to 2D axial acquisitions and to mechanically ventilated animals in order to exploit (1) the global nature of the frequency shift over the slice of interest (15-17) and (2) reproducible breathing. Although this is a simplified scenario for human studies, it presents new MRT tools to possibly validate bio-heat models and monitor temperature rises engendered by MRI examinations.

\section{MATERIALS AND METHODS}

\section{MRT sequence}

The sequence used throughout this work is a 2D (axial acquisition) gradient recalled echo (GRE) sequence repeated continuously (number of repetitions $N=966$ ) (Fig. 1). The different parameters are $T_{\mathrm{E}}=15 \mathrm{~ms}, T_{\mathrm{R}}=33 \mathrm{~ms}, T_{\mathrm{A}}=34 \mathrm{~min}$, flip angle $=12^{\circ}$, matrix size $=64 \times 64$, resolution $=3 \times 3 \times 5 \mathrm{~mm}^{3}$ and bandwidth per pixel $=70 \mathrm{~Hz}$. The peak $10 \mathrm{~g}$ SAR indicated by the scanner with these parameters is $0.4 \mathrm{~W} / \mathrm{kg}$. This was considered sufficiently low to deposit negligible energy in the head of the animal compared with our dedicated heating setup. Each single echo in the sequence is preceded by a 128-point FID acquired just after each selective RF pulse. A linear fit of the phase of the FID versus time then returns the average $\Delta B_{0}$ over the selected slice in the frame rotating at the carrier frequency. As motivated by the model described in (18), and verified experimentally in (15-17), $\Delta B_{0}$ changes induced over the respiration cycle due to chest and diaphragm movement, but also to changes of gas susceptibility, were demonstrated to be global over axial slices to first order, thereby allowing us to correct for this effect by measuring the average $\Delta B_{0}$ throughout the breathing cycle.

The experiment made up of these 966 axial acquisitions is decomposed into three stages. The temporal behavior of $\Delta B_{0}$ over the axial slice is characterized during a first preheating period ( $8 \mathrm{~min}$ ) and extrapolated for the rest of the experiment, which includes a second period with RF heating ( $14 \mathrm{~min}$ ) and a third period aiming at observing the system cooling down, i.e. without RF heating (12 min). The $\Delta B_{0}$ field correction then is applied to the phase of each echo to remove only those

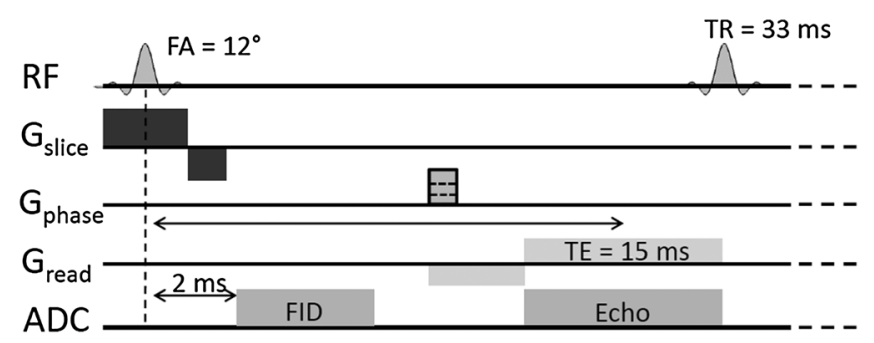

Figure 1. FID navigator-based GRE sequence. Each echo is preceded by an FID whose phase after being unwrapped is fitted with a linear function as a function of time. The slope of the function returns an average $\Delta B_{0}$, which is used to correct each echo via suitable post-processing (phase rewinding). components related to breathing and field drift by suitable post-processing described later on, leaving unaffected the frequency shift due to heating.

\section{Experimental setup}

To increase the reliability of the results, a dedicated setup for RF heating was developed and is presented here. To induce detectable RF heating of the tissues, a relatively significant amount of RF power is needed, which can heat up the RF coil, detune it and yield unreliable phase measurements. For this reason, the experimental setup shown in Fig. 2, which separates RF heating from the RF used for MRI, was developed. Experiments were performed on a 7T Magnetom scanner (Siemens, Erlangen, Germany) equipped with an AC84 gradient $(80 \mathrm{mT} / \mathrm{m}$ maximum amplitude and $400 \mathrm{mT} / \mathrm{m} / \mathrm{ms}$ maximum slew rate) and using a birdcage coil (Invivo, Gainesville, FL, USA) (16 legs, high pass, $26.5 \mathrm{~cm}$ diameter, $25.5 \mathrm{~cm}$ length) operating at $297 \mathrm{MHz}$ for imaging. The RF heating coil was a Bruker (Bruker BioSpin, Ettlingen, Germany) loop coil resonating at $125 \mathrm{MHz}$, on which the back of the head of the baboon was resting. Attenuation of the birdcage coil at $125 \mathrm{MHz}$ was measured to be $-40 \mathrm{~dB}$ using a network analyzer (ZVA8 Rohde \& Schwarz, Munich, Germany). A $-6.5 \mathrm{dBm}$ signal at $125 \mathrm{MHz}$ was sent from a frequency generator (Agilent, Santa Clara, CA, USA) and amplified by $50 \mathrm{~dB}$ by a Bruker RF amplifier, whose output was routed towards the loop coil through a circulator (Wenteq, Duarte, CA, USA) (passband $113-138 \mathrm{MHz}$, isolation $20 \mathrm{~dB}$ ). Reflection at the coil level was monitored by directing the reflected power through the same circulator and sent via a $20 \mathrm{~dB}$ attenuator to a Rohde \& Schwarz FSU spectrum analyzer. Due to the significant amount of power used for heating, the resonance frequency of the loop coil shifted progressively by about $2 \mathrm{MHz}$ during the $14 \mathrm{~min}$ heating period. Manual adjustment of the frequency thus was performed to maintain a weak power reflection at the coil level. Because RF heating was continuous, two stop-band $(115-135 \mathrm{MHz})$ filters of $17 \mathrm{~dB}$ (home made) and $65 \mathrm{~dB}$ (K \& L Microwave, Salisbury, MD, USA) attenuation were inserted before and after preamplification respectively to prevent any $125-127 \mathrm{MHz}$ left signal from corrupting the data at $297 \mathrm{MHz}$.

\section{Post-processing}

The method proposed here was inspired by the work described in References 16 and 17, initially developed to improve the quality of functional MRI data by removing any global $\Delta B_{0}$ variation on each image through the use of navigator echoes. In this work however, these methods could not be applied directly as they would remove the temperature-rise-induced frequency shift contributions. The first $8 \mathrm{~min}$ monitoring stage without RF heating is hence used to characterize the main global field drift as well as the respiration pattern via the FIDs preceding each echo. The field drift, here assumed to be linear, is fitted with a linear function versus time, which is extrapolated for the rest of the experiment. This extrapolated linear trend is then subtracted from the measured $\Delta B_{0}$ data. With the animal being ventilated with a fixed respiration rate, and after correcting for the linear drift, a Fourier series fit with this frequency, taken as the first harmonic is performed on the same preheating period:

$$
\begin{aligned}
\min _{a_{n}, b_{n}} f\left(a_{n}, b_{n}\right)= & \sum_{j=1}^{N}\left(\sum_{n=0}^{P} a_{n} \cos \left(n \omega_{0} t_{j}\right)\right. \\
& \left.+\sum_{n=1}^{P} b_{n} \sin \left(n \omega_{0} t_{j}\right)-\Delta B_{0, j}\right)^{2}
\end{aligned}
$$




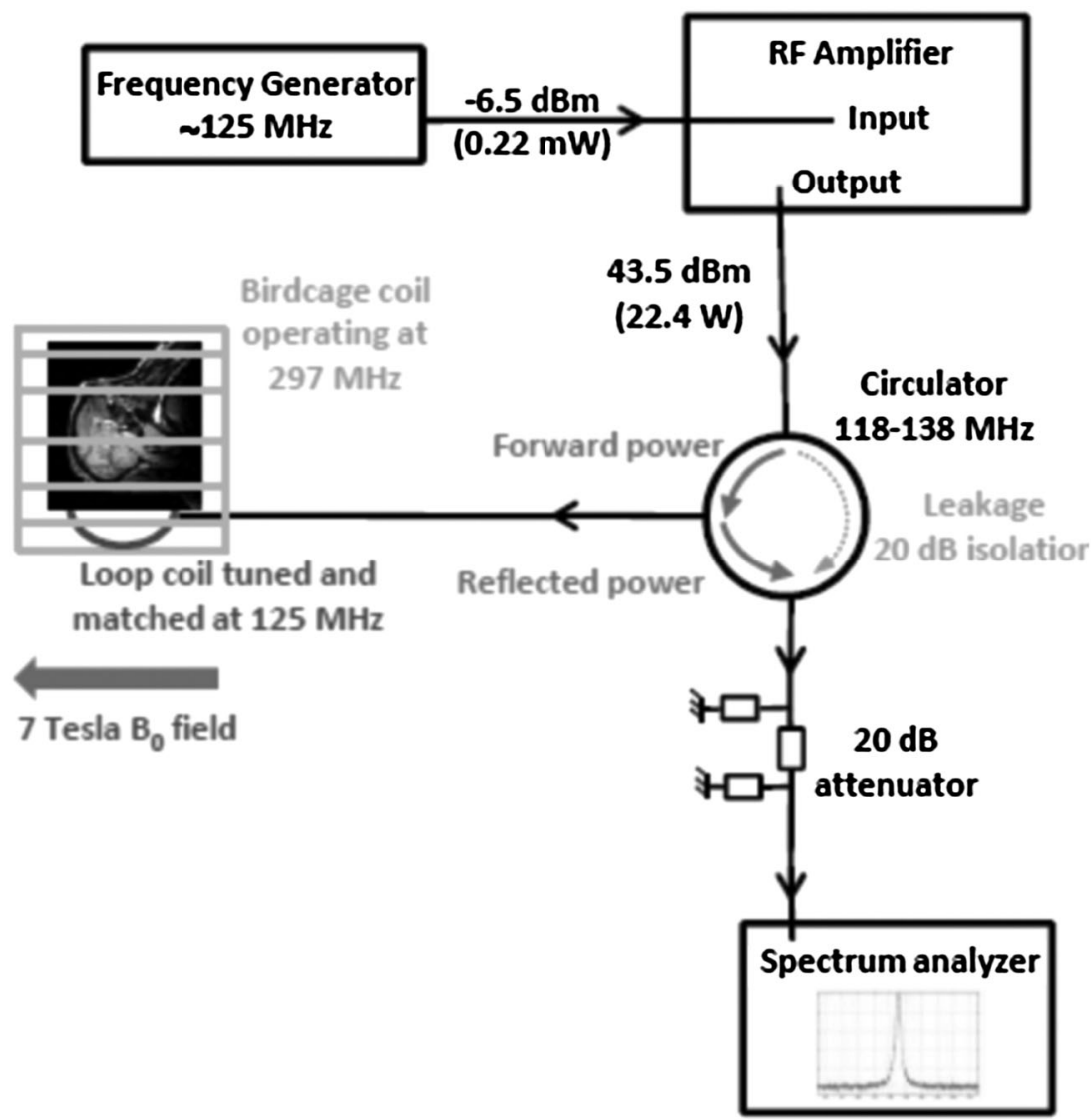

Figure 2. Experimental setup. A $-6.5 \mathrm{dBm}$ signal is generated at $125 \mathrm{MHz}$ and amplified by $50 \mathrm{~dB}$ to reach the loop coil after going through a circulator. Power is reflected back through the same circulator and through a $20 \mathrm{~dB}$ attenuator to be measured on a spectrum analyzer. Detuning of the loop coil occurs due to heating, which is compensated by manual adjustment of the RF frequency. The loop coil is placed below the baboon's head inside the birdcage coil operating at $297 \mathrm{MHz}$.

where $\Delta B_{0, j}$ is the $j$ th sample of the $\Delta B_{0}$ data (one every $\left.T_{\mathrm{R}}=33 \mathrm{~ms}\right), \omega_{0}$ is the angular frequency corresponding to the respiration rate ( 22 cycles/min), $N$ is the number of data points and $P$ is the number of Fourier coefficients. The Fourier coefficients returned via a simple least-squares fit are then kept fixed for the rest of the experiment and characterize breathing alone, without RF-induced temperature changes. Alternatively, these coefficients could be obtained by simple Fourier transformation, although their exact magnitudes and phases may depend on the domain of integration. Throughout the experiment, respiration-induced resonance frequency changes are corrected by optimizing the time lag, which maximizes the cross-correlation between the Fourier series fit above and the newly acquired data (64 echoes acquired for each image). A given sample location in the breathing cycle was therefore attributed to each echo, whose corresponding data were multiplied by $\exp \left(-\mathrm{i} 2 \pi \Delta B_{0} T_{\mathrm{E}}\right) \quad\left(\Delta B_{0}\right.$ being in hertz throughout). Finally, in addition to the global drift mentioned above, reconstruction of the images corresponding to the first stage could still reveal a voxel-dependent linear drift, which was likewise extrapolated for the rest of the experiment to correct for it. The temperature was then obtained by calculating $\left(\varphi-\varphi_{\text {ref }}\right) /\left(2 \pi \alpha v T_{E}\right)$, where $v$ is the Larmor frequency $(297 \mathrm{MHz}), \alpha$ is the frequency shift per degree Celsius $\left(-0.01 \mathrm{ppm} /{ }^{\circ} \mathrm{C}\right), \varphi$ is the phase image and $\varphi_{\text {ref }}$ is the reference phase image taken as the phase of the average of the complex images accumulated during the preheating period. Temperature maps were finally interpolated to match the resolution of an anatomical $T_{1}$-weighted image obtained with a magnetization prepared rapid acquisition GRE sequence $\left(T_{\mathrm{R}}=2.1 \mathrm{~s}, T_{\mathrm{I}}=1.2 \mathrm{~s}\right.$, resolution $\left.=0.94 \times 0.94 \times 1 \mathrm{~mm}^{3}\right)$.

\section{Phantom calibration}

The experiment was first carried out on a $16 \mathrm{~cm}$ diameter spherical phantom in order to roughly assess the SAR to be deposited in the baboon's head as well as the corresponding temperature rise. The phantom was filled with distilled water and doped with $\mathrm{NaCl}(4 \mathrm{~g} / \mathrm{L})$ and agar (1\%), returning measured dielectric properties of $\varepsilon_{\mathrm{r}}=74.6$ and $\sigma=0.78 \mathrm{~S} / \mathrm{m}$ (19). The same 2D GRE sequence with $T_{\mathrm{E}}=25 \mathrm{~ms}$ and $T_{\mathrm{A}}=10 \mathrm{~min}$ was used to perform phase mapping. A fiber optic thermal probe was inserted to measure the $\alpha$ coefficient and establish the correspondence between the resonance frequency and temperature changes in that particular phantom.

\section{Anesthesia}

All animal studies were conducted in accordance with the European convention for animal care and the NIH's Guide for the Care and Use of Laboratory Animals, and were approved by the institutional ethical committee (CETEA protocol 12-059). Experiments were performed on one baboon (male, age 7, weight $30 \mathrm{~kg}$ ). For induction, the animal received an intramuscular injection of ketamine and xylazine $(15 \mathrm{mg} / \mathrm{kg}+1.5 \mathrm{mg} / \mathrm{kg}$ 
respectively). Anesthesia was maintained with sevoflurane (minimum alveolar concentration $\approx 2 \%$ ). The baboon, covered with a blanket, was intubated and mechanically ventilated (Aestiva/5 MRI, General Electrics Healthcare, Fairfield, CT, USA) (tidal volume $8-10 \mathrm{ml} / \mathrm{kg}$, respiration rate $22 / \mathrm{min}$, end-tidal $\mathrm{CO}_{2}$ $38 \mathrm{~mm} \mathrm{Hg}, \mathrm{FiO}_{2}$ 0.5\%). Physiological monitoring (MAGLIFE, Schiller, France) included heart rate, oxygen saturation $\left(\mathrm{SpO}_{2}\right)$, respiratory rate, expired/inspired sevoflurane concentration and end-tidal $\mathrm{CO}_{2}\left(\mathrm{EtCO}_{2}\right)$

\section{RESULTS}

The phantom experiment returned a maximum temperature rise of $1.1^{\circ} \mathrm{C}$ for the $-6.5 \mathrm{dBm}$ RF setting applied for $10 \mathrm{~min}$ (see Fig. 3). The coefficient $\alpha$ was determined to be equal to

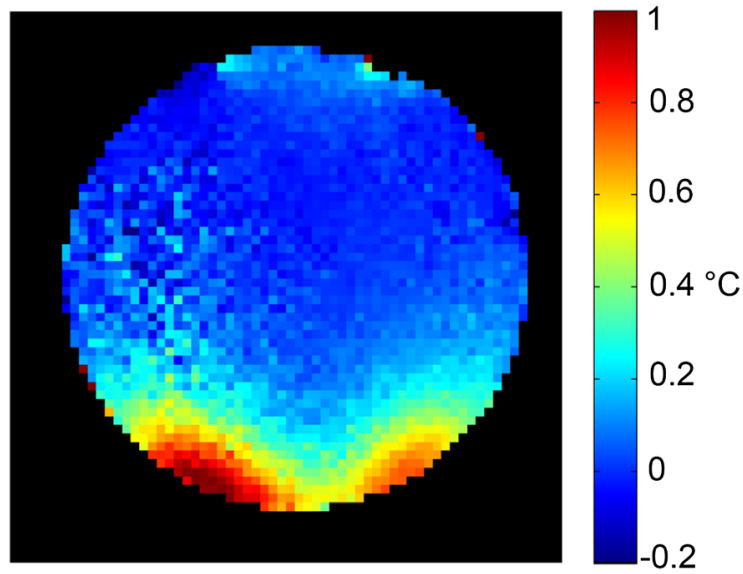

Figure 3. Phantom measurement results. The peak temperature rise reaches $1.1^{\circ} \mathrm{C}$ after $10 \mathrm{~min}$ of RF heating.
$-0.013 \mathrm{ppm} /{ }^{\circ} \mathrm{C}$ by correlating the phase measurements with the temperature recorded by the fiber optic probe. Taking the slope at the origin where maximum temperature rise occurred returned a peak SAR of around $10 \mathrm{~W} / \mathrm{kg}$. For the baboon, the mean $\Delta B_{0}$ data over the imaged axial slice obtained via the FIDs are provided in Fig. 4. In Fig. 4(a), one can see the raw mean $\Delta B_{0}$ data over the slice of interest without any post-processing, one value being obtained every $T_{\mathrm{R}}=33 \mathrm{~ms}$. The first $8 \mathrm{~min}$, where no RF heat source is applied, are used to fit a linear drift versus time (gray line), which is extrapolated for the rest of the experiment and subtracted from the data (Fig. 4(b)). A negative shift of the mean resonance frequency occurs due to heating and returns to baseline $12 \mathrm{~min}$ after the RF heat source is stopped. As an aside, a Fourier transform applied on the data acquired for the first $8 \mathrm{~min}$ reveals different peaks (Fig. 4(c)) corresponding to breathing (first harmonic at 22 cycles/min), eddy currents due to the phase encoding gradient ( 28.4 cycles/min) and heart beat ( 100 cycles/min). While an unexplained and nonnegligible peak at 80 cycles/min remains, the data thus indicate the importance of breathing compared with other noise sources and is in agreement with the values returned by the monitoring equipment. Fig. $4(\mathrm{~d})$ shows a zoom over the $\Delta B_{0}$ data in addition to the fit obtained by minimizing the Euclidean norm of the difference between the data and a Fourier series (20 coefficients) with first harmonic equal to 21.995 cycles/min (root mean square error $=0.18 \mathrm{~Hz}$ ). This fitted waveform is then considered to characterize breathing alone and is used to correct for its variations throughout by optimizing the time lag, which maximizes its cross-correlation with each series of $\Delta B_{0}$ values corresponding to the 64 echoes needed to reconstruct each image.

Figure 5 provides the in vivo temperature results. Figure 5(a) shows an overlay of the anatomical image with the reconstructed temperature rise interpolated. The hot spot locations are in good agreement with the phantom data shown in Fig. 3. Maximum temperature rise in vivo reached a value of $2^{\circ} \mathrm{C}$, a a)
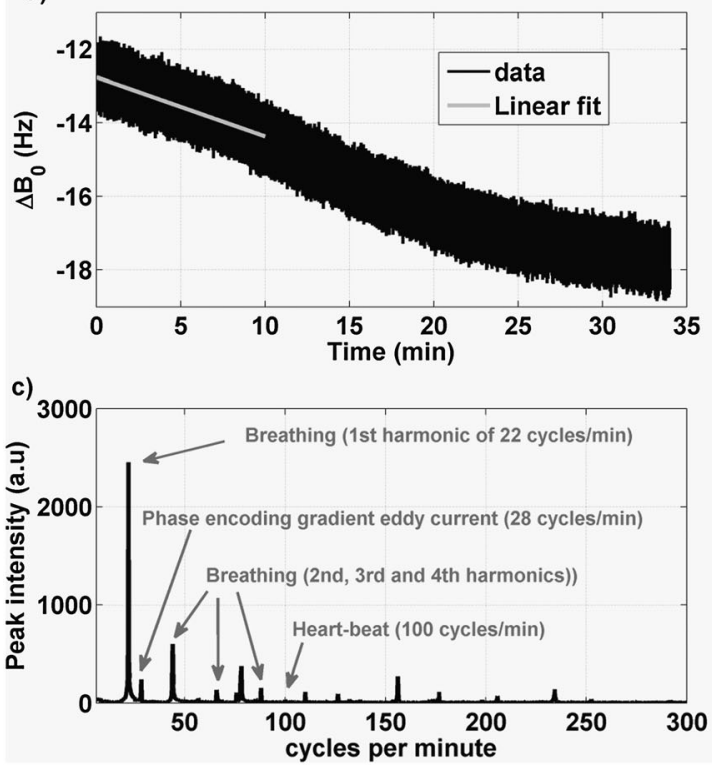

b)
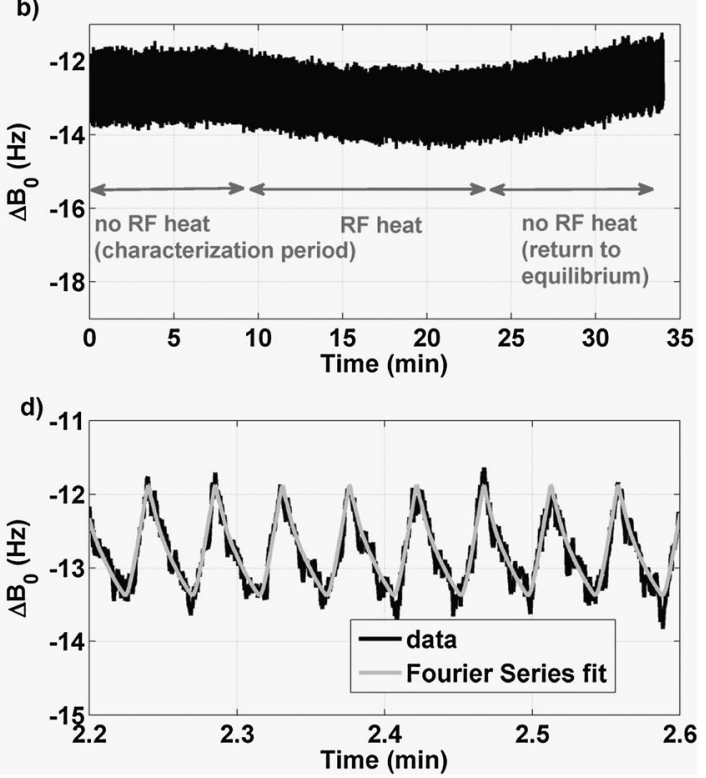

Figure 4. Mean $\Delta B_{0}$ measurements. (a) Raw $\Delta B_{0}$ data (black) versus time and its linear fit (gray) performed during the preheating period. One value of $\Delta B_{0}$ is obtained from each FID, i.e. every $T_{\mathrm{R}}=33 \mathrm{~ms}$. (b) Same data but with correction of the linear drift. (c) Magnitude of the Fourier transform of the data acquired for the first 8 min (after drift correction). (d) Zoomed mean $\Delta B_{0}$ data and its Fourier series fit obtained by minimizing the Euclidean norm of the difference between the data and a Fourier series (20 coefficients) with first harmonic at 21.995 cycles/min. 
a)

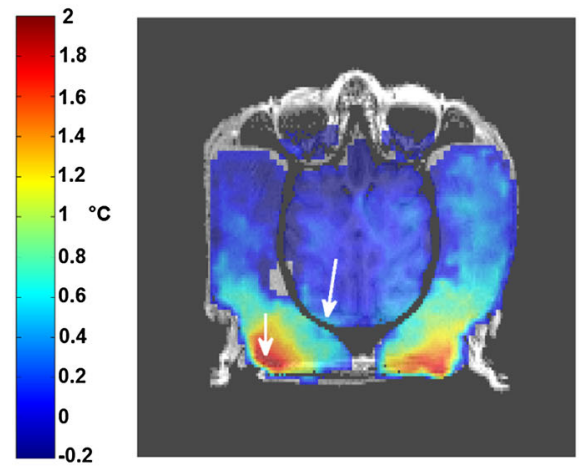

b)

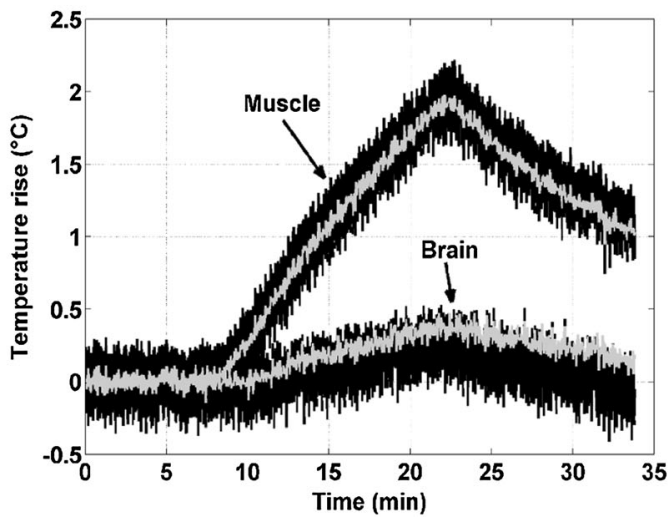

Figure 5. Temperature results: (a) overlay of an anatomical image with the temperature map; (b) temperature rise versus time at the two locations shown in (a) without corrections (black) due to breathing and with corrections (gray). The standard deviations over the first 8 min are 0.05 and $0.2{ }^{\circ} \mathrm{C}$ when the corrections due to breathing are and are not made respectively.

value about twice as high as in the phantom, which can be explained by the closer proximity of the baboon's head surface to the plane of the loop coil and the longer heating time ( $14 \mathrm{~min}$ versus $10 \mathrm{~min}$ ). Fig. $5(\mathrm{~b})$ shows the temperature timecourses at the two points indicated in Fig. 5(a), corresponding to muscle and brain regions, with (gray) and without (black) corrections due to breathing respectively. The proposed monitoring and correction reduces the standard deviation of the data over the first $8 \mathrm{~min}$ from $0.2^{\circ} \mathrm{C}$ to $0.05^{\circ} \mathrm{C}$. After RF heating was stopped, and when the last period was sufficiently long, the temperature roughly went back to the baseline value, thereby providing further confidence in the extrapolation made throughout (see also Fig. 4(b)).

\section{DISCUSSION}

We have reported a new method for monitoring small temperature changes in the brain of ventilated large animals. Experimental results were obtained with a 7T clinical scanner on an anesthetized baboon, revealing a precision of less than $0.1^{\circ} \mathrm{C}$. Yet the real accuracy and precision depend on the exact value of the $\alpha$ coefficient, here taken as equal to $-0.01 \mathrm{ppm} /{ }^{\circ} \mathrm{C}$, which in fact can vary by $\pm 20 \%$ due to susceptibility effects (2). Additional and independent measurements would be needed to confirm the accuracy of the method. Overall, the method relies on a first period stage

- where breathing, here assumed to be reproducible, is modelled via an original Fourier analysis of the time course of phase fluctuations measured through repeated FID signals and

- where the main field low frequency drift is fitted with a linear function versus time and extrapolated.

Although the drift in general can be nonlinear in time, the measurement reported in Fig. 4(a) suggests a linear behavior, at least during the preheating stage. Extrapolating this behavior for the rest of the experiment therefore seems reasonable, as long as the perturbation is small and the experiment is not too long. The return to baseline after the external RF heat source is stopped validates to some extent this extrapolation.

Developing the dedicated setup (Fig. 2) appeared to be a necessity to interfere as little as possible with the MRI equipment, which, if it is too disturbed, influences the measured phase.
Furthermore, it was crucial to insert stop-band filters to remove any $125 \mathrm{MHz}$ signal, despite the intrinsic attenuation of the birdcage coil at this frequency. Without these filters, the $125 \mathrm{MHz}$ signal would corrupt the $297 \mathrm{MHz}$ signal and cause sudden $\Delta B_{0}$ jumps in the FIDs whenever the external RF source was turned on and off.

The source of the drift can have several origins, including fluctuations of the room temperature, change of the animal's internal temperature, cryogen boiloff and gradient heating (20). In this work, not monitoring the animal internal temperature was clearly a weakness. A temperature probe with sufficient cable length and compatible at $7 \mathrm{~T}$ was not however available in our laboratory. The animal warming up during the preheating stage (Fig. 4(a)) in any case would be opposite to what commonly occurs during anesthesia, i.e. a drop of temperature (12). Room temperature was also measured with extra sensors both in the magnet and in the receiver-clock room, indicating a fluctuation of less than $0.1^{\circ} \mathrm{C}$ during the entire experiment. Given a possible $0.01 \mathrm{ppm} /{ }^{\circ} \mathrm{C}$ thermal coefficient of the $10 \mathrm{MHz}$ crystal (21), such small variations in the temperature electronics would result in less than a $0.1^{\circ} \mathrm{C}$ temperature noise in the images. Cryogen boiloff and gradient heating are therefore currently the most plausible causes. Due to the linear extrapolation used here, caution would be advised if longer experiments were attempted. As was done here, a last monitoring period, after RF heating is stopped, can be implemented to check some of the assumptions underlying the extrapolation, provided of course the temperature does go back to its initial value, which is not certain. At locations where the temperature rise was insignificant, the global $\Delta B_{0}$ correction applied over the whole slice may also be inaccurate, leading to small negative temperatures (upper part of Fig. 5). The fact that the temperature time course reconstructed in the brain after correction (gray line in Fig. 4(b)) is not vertically centered in the data without correction may indeed reveal at this particular location a small deviation from this model (15). Other methods have been proposed to monitor both the drift and respiration fluctuations via the phase measured in voxels containing fat (22). While this is certainly appealing due to its simplicity and since no extrapolation would be needed, it remains also an approximation given that the susceptibility temperature-induced change in fat leads to a frequency shift comparable with the one due to chemical shift change in water (23). While fatty tissue has a very small conductivity 
( $\sim 0.04 \mathrm{~S} / \mathrm{m}$ at $300 \mathrm{MHz}$ ), it can still warm up via thermal diffusion. This method therefore would require either small time scales so that barely any thermal diffusion could take place or clearly identified voxels experiencing no temperature change. Lastly, collecting the data needed to reconstruct a phase image for phase correction would either force a loss of temporal resolution or require the use of fast acquisition methods such as echo planar imaging (22), likely adding thermal and mechanical stress to the gradients and magnet bore. Monitoring temperature by looking in the voxels containing fat thus remains the topic of further studies. Field cameras (24) should also be a promising technology to monitor the global as well as other low order spatial shifts of the resonance frequency, thereby increasing the accuracy of the measurements, but at the cost of more hardware complexity.

Future work also involves looking more closely at the correspondence between the experimental data and predictions given by Pennes' bioheat model (14), or other suggested models (12). Heat diffusion obviously complicates the matter and requires the full numerical integration of the corresponding partial differential equation. Following Pennes' model, neglecting heat diffusion and despite the possible effects of anesthesia on perfusion (25), one could have hoped to observe in Fig. 5(b) a behavior of the form $T(t)=T_{\max }\left(1-\mathrm{e}^{-B t /\left(\rho C_{p}\right)}\right)$ (B is the perfusion term in $\mathrm{W} / \mathrm{m}^{3} / \mathrm{K}, \rho$ is the density in $\mathrm{kg} / \mathrm{m}^{3}, C_{p}$ is the specific heat capacity in $\mathrm{J} / \mathrm{kg} / \mathrm{K}$ and $T_{\max }=\rho \mathrm{SAR} / B$ ), i.e. an equilibrium between the SAR and perfusion terms with typical timescale on the order of a few minutes in white matter. The absence of such a plateau in the brain (Fig. 5(b)) however does not contradict Pennes' model, because heating in this region mainly occurred due to heat diffusion, as shown by the apparent zero initial slope in the corresponding curve, when RF heating starts (see Fig. 5(b)). As far as the muscle is concerned, the value of perfusion reported in the literature is too small (11) to expect a clear curvature over $14 \mathrm{~min}$, and diffusion likewise plays a role. Significantly more RF power, or simply a different SAR distribution where a higher energy deposit would occur further inside the brain, would be needed to better compare the measurements with the predictions made by Pennes' model in the brain, at least qualitatively. However, first our RF amplifier was at its maximum capacity, and second more power would induce accordingly more temperature rise in the muscle, which ruled out this option. For this purpose, as shown in (26), the use of volume coils, or perhaps more simply Helmholtz coils, could be more appropriate to distribute the RF energy more deeply in the brain.

The applicability of this method to other species also remains the topic of further studies. For smaller animals, at possibly higher fields, the global $\Delta B_{0}$ offset model for axial slices described in (18) may become inaccurate, depending on the distance/geometry of the lungs with respect to the brain. For humans, preliminary tests have shown that in the absence of anesthesia and due to motion the $\Delta B_{0}$ time-course could easily become erratic and almost impossible to extrapolate. The method as currently described in this work therefore could hardly be applied in this scenario. Perhaps short periods of apnea could be performed to mitigate the breathing effects, while the FID data could still reveal the mean $\Delta B_{0}$ value for each of these periods. Spectroscopic measurements also could potentially overcome these problems and provide an evaluation of the absolute temperature by looking at the difference between the water peak and some reference peak insensitive to temperature $(27,28)$, but at the expense of spatial and temporal resolution.

\section{Acknowledgements}

The authors thank David Brunner and Béchir Jarraya for valuable discussions.

\section{REFERENCES}

1. Hindman JC. Proton resonance shift of water in gas and liquid states. J. Chem. Phys. 1966; 44: 4582-4592.

2. Rieke $V$ and Pauly KB. MR thermometry. J. Magn. Reson. Imaging 2008; 27: 376-390.

3. Bradley WG Jr. J. Am. Coll. Radiol. 2009; 6(7): 510-513. DOI: 10.1016/j. jacr.2009.01.004

4. Pauly KB, Diederich CJ, Rieke V, Bouley D, Chen J, Nau WH, Ross AB, Kinsey AM, Sommer G. Magnetic resonance-guided high-intensity ultrasound ablation of the prostate. Magn. Reson. Imaging 2006; 17: 195-207.

5. McDannold N, Tempany CM, Fennessy FM, So MJ, Rybicki FJ, Stewart EA, Jolesz FA, Hynynen K. Uterine leiomyomas: MR imaging-based thermometry and thermal dosimetry during focused ultrasound thermal ablation. Radiology 2006; 240: 263-272.

6. Jolesz FA, Hynynen K. Magnetic resonance image-guided focused ultrasound surgery. Cancer J. 2002; 8 (Suppl. 1): S100-112.

7. de Senneville BD, Quesson B, Moonen CT. Magnetic resonance temperature imaging. Int. J. Hyperthermia 2005; 21: 515-531.

8. Salomir R, Viallon M, Kickhefel A, Roland J, Morel DR, Petrusca L, Auboiroux V, Goget T, Terraz S, Becker CD, Gross P. Reference-free PRFS MR-thermometry using near-harmonic 2-D reconstruction of the background phase. IEEE Trans. Med. Imaging 2012; 31: 287-301.

9. Grissom WA, Lustig M, Holbrook AB, Rieke V, Pauly JM, Butts-Pauly K. Reweighted $\ell_{1}$ referenceless PRF shift thermometry. Magn. Reson. Med. 2010; 64: 1068-1077.

10. Wang Z, Lin JC, Mao W, Liu W, Smith MB, Collins CM. SAR and temperature: simulations and comparison to regulatory limits for MRI. J. Magn. Reson. Imaging 2007; 26: 437-441.

11. Massire A, Cloos MA, Luong M, Amadon A, Vignaud A, Wiggins CJ, Boulant $\mathrm{N}$. Thermal simulations in the human head for high field MRI using parallel transmission. J. Magn. Reson. Imaging 2012; 35: 1312-1321.

12. Shrivastava D, Hanson T, Kulesa J, Tian J, Adriany G, Vaughan JT. Radiofrequency heating in porcine models with a 'large' $32 \mathrm{~cm}$ internal diameter, 7T (296 MHz) head coil. Magn. Reson. Med. 2011; 66: 255-263.

13. Boulant N, Massire A, Amadon A, Vignaud A. Radiofrequency pulse design in parallel transmission and under strict temperature constraints. Magn. Reson. Med. 2013. DOI: 10.1002/mrm.24974.

14. Pennes HH. Analysis of tissue and arterial blood temperatures in the resting human forearm. J. Appl. Physiol. 1948; 1: 93-122.

15. Van de Moortele P-F, Pfeuffer J, Glover GH, Ugurbil K, Hu X. Respiration-induced $B_{0}$ fluctuations and their spatial distribution in the human brain at 7 Tesla. Magn. Reson. Med. 2002; 47: 888-895.

16. Pfeuffer J, Van de Moortele P-F, Ugurbil K, Hu X, Glover GH. Correction of physiologically induced global off-resonance effects in dynamic echo-planar and spiral functional imaging. Magn. Reson. Med. 2002; 47: 344-353.

17. Hu X, Kim SG. Reduction of signal fluctuation in functional MRI using navigator echoes. Magn. Reson. Med. 1994; 31: 495-503.

18. Raj D, Paley DP, Anderson AW, Kennan RP, Gore JC. A model for susceptibility artefacts from respiration in functional echo-planar magnetic resonance imaging. Phys. Med. Biol. 2000; 45: 3809-3820.

19. Nicolson A, Ross G. Measurement of the intrinsic properties of materials by time-domain techniques. IEEE Trans. Instrum. Meas. 1970; 19: 377-382.

20. Peters RD, Hinks RS, Henkelman RM. Ex vivo tissue-type independence in proton-resonance frequency shift MR thermometry. Magn. Reson. Med. 1998; 40: 454-459.

21. Peters RD. Magnetic Resonance Thermometry for Image Guided Thermal Therapy. University of Toronto. PhD Thesis, 2000.

22. Streicher MN, Schäfer $A$, Ivanov $D$, Muller DK, Amadon A, Reimer $E$, Huber L, Dhital B, Rivera D, Kogler C, Trampel R, Pampel A, Turner R. Fast accurate MR thermometry using phase referenced asymmetric spin-echo EPI at high field. Magn. Reson. Med. 2014; 71: 524-533.

23. Sprinkhuizen SM, Maurits K, Konings MK, van der Bom MJ, Viergever MA, Bakker CJG, Bartels LW. Temperature-induced tissue 
susceptibility changes lead to significant temperature errors in PRFSbased MR thermometry during thermal interventions. Magn. Reson. Med. 2010; 64: 1360-1372.

24. Barmet C, De Zanche N, Pruessmann KP. Spatiotemporal magnetic field monitoring for MR. Magn. Reson. Med. 2008; 60: 187-197.

25. Kaisti KK, Metsähonkala L, Teräs M. Effects of surgical levels of propofol and sevoflurane anesthesia on cerebral blood flow in healthy subjects studied with positron emission tomography. Anesthesiology 2002; 96: 1358-1370.

26. Boulant N, Katscher U, Luong M, Massire A, Amadon A, Vignaud A. B1+-based SAR assessment using a birdcage coil at 7 Tesla: experimental evaluation using magnetic resonance thermometry. In: Proceedings of the 22nd Annual Meeting of the ISMRM, Milan, Italy. ISMRM: Berkeley CA, p. 1987.

27. Cady EB, D'Souza PC, Penrice J, Lorek A. The estimation of local brain temperature by in vivo $1 \mathrm{H}$ magnetic resonance spectroscopy. Magn. Reson. Med. 1995; 33: 862-867.

28. Marshall I, Karaszeski B, Wardlaw JM, Cvoro V, Wartolowska K, Armitage PA, Carpenter T, Bastin ME, Farrall A, Haga K. Measurement of regional brain temperature using proton spectroscopic imaging: validation and application to acute ischemic stroke. Magn. Reson. Imaging 2006; 24: 699-706. 